\title{
Imaging of Endometriosis: The Role of Ultrasound and Magnetic Resonance
}

\author{
Valentina Testini ${ }^{1,2} \cdot$ Laura Eusebi $^{3}$ - Gianluca Grechi ${ }^{4} \cdot$ Francesco Bartelli $^{3}$. \\ Giuseppe Guglielmi ${ }^{1,2,5}$ (B)
}

Accepted: 7 February 2022/Published online: 18 February 2022

(C) The Author(s) 2022

\begin{abstract}
Endometriosis is a chronic gynecological disease characterized by the growth of functional ectopic endometrial glands and stroma outside the uterus. It causes pelvic pain, dysmenorrhea, dyspareunia, or infertility. Diagnosis requires a combination of clinical history, noninvasive and invasive techniques. The aim of the present review was to evaluate the contribution of imaging techniques, mainly transvaginal sonography and magnetic resonance imaging to diagnose different locations and for the most appropriate treatment planning. Endometriosis requires a multidisciplinary teamwork to manage these patients clinically and surgically.
\end{abstract}

Keywords Endometriosis · Deep endometriosis · Endometrioma · Transvaginal ultrasonography $\cdot$ MRI

This article is part of the Topical collection on Global Radiology.

Giuseppe Guglielmi

giuseppe.guglielmi@unifg.it

1 Radiology Unit, “Dimiccoli”' Hospital, Viale Ippocrate 15, 70051 Barletta, Italy

2 Department of Clinical and Experimental Medicine, Foggia University School of Medicine, Viale L. Pinto 1, 71121 Foggia, Italy

3 Radiology Unit, "Carlo Urbani”" Hospital, Jesi, Italy

4 Obstetrics and Gynecology Unit, "Carlo Urbani”' Hospital, Jesi, Italy

5 Radiology Unit, Hospital "Casa Sollievo Della Sofferenza”, Viale Cappuccini 2, 71013 San Giovanni Rotondo, Foggia, Italy

\section{Introduction}

Endometriosis is a common gynecological inflammatory condition that is defined as functional ectopic endometrial glands and stroma outside the uterus. This disease affects women of reproductive age, with a prevalence of approximately $10 \%$ [1]. Patients can be asymptomatic or present with chronic pelvic pain and/or infertility.

The phenotypes of endometriotic lesions can be divided into three groups: pelvic endometriosis, ovarian endometriomas (OMA) and deep infiltrating endometriosis (DIE) [2]. In particular, pelvic endometriosis is defined as the presence of any endometrial tissue within the pelvic cavity, including the peritoneum, within any of the pelvic organs and inside the pouch of Douglas (POD). Ovarian endometriosis, an endometrioma, is defined as an ovarian cyst, of different size, lined by endometrial tissue. DIE is defined as endometriotic tissue that penetrates the retroperitoneal space for a distance of $5 \mathrm{~mm}$ or more and may be present in multiple locations, involving anterior or posterior pelvic compartments, or both [3]. Posterior DIE, a multifocal disease that may affect a variety of anatomical sites, represents the most common type of DIE [4]. The most typical sites of DIE include uterosacral ligaments (USL), rectovaginal septum (RVS), vaginal wall, POD and bowel, predominantly below the rectosigmoid junction. Anterior DIE corresponds to disease involving the anterior pouch or bladder and is much less common. DIE is a source of pain and infertility [5].

A frequently association with endometriosis is represented by adenomyosis, a disease characterized by infiltration of endometrial tissue into the myometrium [6]. 


\section{Etiopathogenesis}

Although the pathogenesis of endometriosis has not been fully elucidated, it is commonly thought that endometriosis occurs when endometrial tissue contained within menstrual fluid flows retrogradely through the fallopian tubes and implants at an ectopic site within the pelvic cavity [7]. In this process, menses transports viable endometrial fragments through the fallopian tubes to the peritoneal cavity, where they are able to implant, develop and sometimes invade other tissues of the pelvis [8]. In favor of this hypothesis is that all known factors that increase menstrual flow are also risk factors for endometriosis, including early age at menarche, heavy and long periods as well as short menstrual cycles [9]. The anatomical distribution of endometriotic lesions can also be explained by the hypothesis of retrograde menstruation as endometriotic lesions tend to have an asymmetrical distribution, which could be explained by the effect of gravity on menstrual flow, the abdominopelvic anatomy and the peritoneal clockwise flow of menses [10]. However, this theory does not explain the fact that although retrograde menstruation is seen in up to $90 \%$ of women, only $10 \%$ of women develop endometriosis [3]. Moreover retrograde menstruation does not explain the mechanism of endometrial tissue grafting onto the peritoneum. It is therefore evident that a variety of environmental, immunological and hormonal factors contribute to the onset of endometriosis, with mechanisms not yet known [11].

Genetic factors play an important role in the genesis of endometriosis, with an up to six times greater risk of developing the disease for first degree relatives of patients with endometriosis [12]. Despite this clear inheritance, the identification of the genetic factors that drive the disease is still incomplete.

\section{Diagnosis}

The first step in diagnosing DE is to establish the patient's clinical history with particular emphasis on symptoms (dysmenorrhea, dyspareunia, dysuria, dyschezia, and chronic pelvic pain) as well as, age, height, weight, ethnic origin, gravidity, parity, previous surgery for endometriosis, family history of endometriosis, previous non-surgical treatment for endometriosis, and infertility. No symptom is specific to endometriosis [13].

However, several authors have underlined the poor relationship between symptoms exhibited by patients and the severity of the lesions rendering clinical diagnosis difficult. Moreover, it is thought that up to $50 \%$ of women could have asymptomatic endometriosis $[14,15]$.
The second step is based on physical examination including a systematic analysis of the posterior vaginal fornix with a speculum to look for retraction and dark nodules. Digital examinations should be performed of the vagina to assess the characteristics of the uterus and adnexa, of the vesicouterine pouch to detect bladder invasion, and of the retrocervical area to detect infiltration of the torus uterinus, uterosacral ligaments (USLs), pouch of Douglas (POD), vagina, and rectovaginal septum (RVS) [13]. Rectal digital examination can help in assessing the involvement of the rectum, parametrium and visceral pelvic fascia. In the particular setting of DE, few data are available to evaluate the accuracy of physical examination. One retrospective study found that routine clinical examination detected DE in only $36 \%$ of 140 women with DE, and the authors suggest the accuracy of physical examination improves during menstruation [16]. To detect rectosigmoid and retrocervical DE without differentiating between the different specific DE locations, Abrao et al. reported that digital vaginal examination had a sensitivity of $72 \%$ and $68 \%$, a specificity of $54 \%$ and $46 \%$, respectively [17].

Laboratory tests are limited in the diagnosis of endometriosis (CA-125 has a detection rate of only $54 \%$ in patients with severe endometriosis 5 and is neither sensitive nor specific for the diagnosis) [18].

The gold standard for diagnosis of endometriosis is based on laparoscopy or surgery with histological verification of endometrial glands and/or stroma.

Imaging is needed to diagnose endometriosis and to plan treatment. The techniques used are transvaginal ultrasound (TVUS) and magnetic resonance imaging (MRI), the latter should be considered as a second-line technique after ultrasound [1].

\section{Treatment}

Treatment of endometriosis is complicated and involves conservative approaches combined with medical therapies or surgery. Imaging is crucial to guide the type of treatment. The American Society for Reproductive Medicine Practice Committee states that "endometriosis should be viewed as a chronic disease that requires a lifelong management plan with the goal of maximizing the use of medical treatment and avoiding repeat surgical procedures" [18]. Current treatment is essentially surgical, medical, or a combination of both approaches. To this end, many patients are stratified for medical treatment with or without surgical treatment based on symptom severity or imaging results and desire to have children, with medical therapy typically including non-steroidal anti-inflammatory drugs, oral contraceptives, androgens, progestogens and 
gonadotropin-releasing hormone $(\mathrm{GnRH})$ and/or surgery [19].

Laparoscopy is an effective surgical approach with the goal of excision of visible endometriosis in a hemostatic fashion. Radical surgery is reserved for those patients with severe symptoms where there is no desired fertility potential and especially when other forms of treatment have failed. Total abdominal hysterectomy and bilateral salpingo-oophorectomy are performed along with resection of any endometriotic lesions as completely as possible [4].

\section{Ultrasound}

Transvaginal ultrasound (TVUS) is typically the initial imaging evaluation performed in patients with pelvic pain and infertility or when there is clinical suspicion for endometriosis. This examination is widely available with low relative cost and the sensitivity and specificity for detection of ovarian endometriomas and lesions in the rectal wall are high [18].

All patients should be examined systematically and carefully using an endocavitary sonography, with a microconvex array probe inserted transvaginally or transrectally. Both techniques are optimal approaches for examining uterus (including the different uterine zones: cervix, endometrium, junctional zone, and myometrium), adnexa, paracolpium, parametrium, vesicocervical, vesicovaginal, and rectovaginal spaces as well as urinary bladder, ureters, and rectum [20].

Limitations of sonographic technique include anterior compartment detection of endometriosis (bladder and vesicouterine pouch detection) and detection within the middle compartment (torus uterinus and round ligaments) [18].

The pelvic localization of endometriosis can be described according to three compartments (central, anterior, and posterior) according to functional and clinical relevance [21]. The anterior compartment includes the insertion site of the ureters, the bladder, the vesicouterine pouch, and the vesicovaginal pouch. The middle compartment contains the uterine body, fallopian tube, and uterine ligaments. The posterior compartment contains the uterosacral ligaments, rectovaginal septum, anterior rectal wall, and sigmoid colon [21].

Recently, the IDEA (International Deep Endometriosis Analysis group) published a consensus report 18 on the appropriate terms, definitions, and measurements that may be used to describe the sonographic features of the different phenotypes of endometriosis. A standardized pelvic TVS approach is proposed by this IDEA report consisting in four step pelvic evaluation [22]:
(1) Evaluation of uterus and ovaries:

(a) Evaluation of uterus: 2D-3D sonographic signs of adenomyosis

(b) Evaluation of the adnexa: presence or absence of endometrioma or tubal pathology

(2) Evaluation of TVS organ mobility (adhesions): adnexa and uterine mobility site-specific tenderness

(3) Pouch of Douglas (POD) assessment using real-time ultrasound-based "sliding sign"

(4) Assessment for DIE nodules in anterior lateral and posterior compartments

\section{Anterior Compartment}

The anterior compartment is comprised of the urinary bladder, the vesicouterine pouch, round ligaments and ureters. Involvement of the urinary tract occurs in approximately $1-2 \%$ of patients with endometriosis and involves bladder in $85 \%$ of these cases [23]. Ureteric involvement is found in $4 \%$ of patients with rectovaginal endometriosis [24]. The prevalence of round ligament endometriosis is estimated between $4.3 \%$ and $13.8 \%$ [25].

Bladder endometriosis is considered only in case of infiltration of the bladder wall and not in case of adhesions or superficial peritoneal implants on the bladder serosa.

Before TVS scan, patients are asked not to empty completely the bladder, because the slightly filled bladder permits to better evaluate the structure of the walls.

On ultrasound, bladder endometriosis appears as hypoechoic lesion, either containing cystic lesions or not, with regular/irregular margins of the bladder wall, bulging toward the lumen, involving the serosa, muscularis (most common), or (sub)mucosa of the bladder [26].

In the assessment of the bladder DIE localization, the bladder wall can be divided into three zones: the trigonal zone and vesical base; the vesical dome (which lies superior to the trigone and is intra-abdominal) and the anterior retroperitoneal bladder. Most frequently bladder endometriosis is located in the vesical dome on the posterior bladder wall close to the vesicouterine pouch [27].

Bladder adhesions of the vesicouterine pouch are evaluated by the presence or absence of the "sliding sign" between the uterus and the bladder [28].

During examination, from a longitudinal section through the cervix and moving the probe toward the lateral pelvic wall, it is possible to assess the distal part of the ureter adjacent to the bladder trigone, in order to evaluate the presence of stenosis and subsequent cephalad dilatation of the pelvic ureters. This finding can suggest direct invasion 
or compression of the ureter by endometriotic nodules, ovarian endometriomas, or adhesions [28].

The prevalence of ureteral endometriosis ranges from 0.01 to $1 \%$ of all patients with the disease and most often affect the distal segment of the ureter [29]. There are two types of endometriosis involvement of ureters: (1) extrinsic that represents $75-80 \%$ of the cases and is defined as the presence of endometrial tissue in the outer adventitia of the ureter that occurs as a nodule encasing the ureter by extension from pelvic foci; (2) intrinsic that represents $20-25 \%$ of cases and is defined as the presence of endometrial tissue in the mucosal and/or muscular layer of the ureter. Imaging signs are nodule or mass occurring in the ureter along its course, dilatation of the pelvic ureteral tract, or ureteropelvic hydronephrosis superior to the suspected lesion [22]. Pelvic ureteral dilation can be easily seen by TVS as a tubular anechoic image, very similar to a blood vessel but with negative color/power Doppler signs. An extrinsic compression, also without ureteral dilatation, is suspected in cases where a DIE lesion is located close to the ureter. The observation of a possible ureteral involvement requires transabdominal ultrasound to evaluate the renal pelvis. In all women with DIE, a transabdominal scan of the kidney to search for ureteral stenosis is necessary because the prevalence of endometriotic lesions in the urinary tract may be underestimated and women with DIE involving the ureter may be asymptomatic [30].

A review of the literature for bladder endometriosis reveals a reported mean US sensitivity of $55 \%$ and specificity of $93.5 \%$ [31].

For vesicouterine pouch endometriosis, two series reported US sensitivities and specificities of $16.7 \%$ and $33 \%$ and $99 \%$ and $100 \%$ [32]. These discrepant results could be partly explained by selection bias of inclusion among studies.

\section{Central Compartment}

The central compartment includes uterus and adnexa.

\section{Uterus}

Adenomyosis is characterized by the migration and proliferation of endometrial glands and stroma from the basal layer of endometrium into the myometrium. It is associated with smooth muscle hyperplasia leading to an ultrasound image of ill-defined lesions within the myometrium [22].

Recently, the ultrasound features have been systematically described by the international Morphological Uterus Sonographic Assessment (MUSA) group [33].

According to the MUSA consensus, adenomyosis should be described as localized and diffuse.
Adenomyosis is classified as diffuse, if the total involvement of myometrium exceeds $50 \%$ of the corpus uteri (when the findings are present in only one part of the myometrium on one or more sites within the uterine wall), and localized (or focal) when less than $50 \%$ of myometrium is involved (one or more lesions) [33]. An adenomyoma is defined as a focal consolidation of endometrial glands and or endometrial stroma located within the myometrium with additional compensatory hypertrophy of the surrounding myometrium [27]. In rare cases it may present as a large cyst (adenomyotic cyst or cystic adenomyoma, with largest diameter $2 \mathrm{~mm}$ and echogenic rim) [34].

To evaluate adenomyosis, the size of the lesions should be measured (in particular the largest diameter of each focal lesion or the myometrial wall thickness in cases of a diffuse lesion), the involvement of the uterine layers and of the extent of the disease, based on the estimated volume of the uterine corpus affected by adenomyosis (mild $<25 \%$, moderate $25-50 \%$, and severe $>50 \%$ ) $[27,35]$.

According to several studies, there are different features associated with adenomyosis visible on $2 \mathrm{D}$ transvaginal sonographic [33].

On ultrasound, an adenomyotic uterus appears with a globular shape, enlarged dimensions, and uterine wall asymmetry. The myometrium typically appears inhomogeneous on gray-scale, characterized by the presence of an indistinctly defined area with either decreased or increased echogenicity with myometrial hypoechoic linear striations [36]. Round anechoic areas of $1-\mathrm{mm}$ to $7-\mathrm{mm}$ diameter, named myometrial cysts, also could be present within the myometrium [36]. In cases of focal adenomyosis, the adenomyotic lesion appears as a heterogeneous and hypoechogenic area within the myometrium, usually with anechoic lacunae or cysts with ill-defined contours and fanshaped shadowing. These hypoechogenic areas reflect muscular hypertrophy of the myometrial tissue [27]. Irregularities of the endometrial-myometrial junctional zone is another common ultrasound marker in the diagnosis of adenomyosis [27]. This endomyometrial interface is normally visualized as hypoechoic tissue layer seen beyond the endometrial basal layer. In women with adenomyosis, the diffuse or focal hyperplasia and hypertrophy of myocytes determine whether diffuse or focal thickening of this zone is seen [37].

A characteristic sign is the "question mark sign" defined when the corpus uterus was flexed backward, the fundus of uteri was facing the posterior pelvic compartment, and the cervix was directed frontally toward the urinary bladder [38]. Investigators found $93 \%$ specificity and $75 \%$ sensitivity of this sign in detecting adenomyosis [22].

Power Doppler can be used to distinguish myometrial cysts from blood vessels and discriminate between 
leiomyomas and focal adenomyosis. Uterine leiomyomas manifest a circular flow along the myoma pseudocapsule, while localized adenomyosis and adenomyomas are characterized by diffusely spread vessels inside the lesions [39]. 2D ultrasound can yield equivocal result in the case of focal adenomyosis especially if there are coexistent fibroids. A meta-analysis of 14 trials and 1985 participants reported the sensitivity and specificity of ultrasound in the diagnosis of adenomyosis to be as high as 82.5 and $84.6 \%$, respectively, values in line with MRI values [40].

The use of 3D vaginal ultrasound for the diagnosis of adenomyotic pathology allows a more complete evaluation in the sagittal, transverse and coronal planes, evaluating the ultrasound signs on the acquired 3D volume of the uterus [41].

\section{Ovaries}

Ovarian endometriomas occur when ectopic endometrial tissue in the ovary hemorrhages, forms a hematoma, enveloped by ovarian parenchyma (Fig. 1).

An ovarian endometrioma has different imaging appearances on US, with the classic appearance being a cyst unilocular or multilocular (less than five locules) with homogeneous low-level echogenicity (ground glass echogenicity) of the cyst fluid, with increased posterior through transmission and no vascularization on color Doppler [22] (Fig. 2).

Another feature is the presence of peripheral echogenic foci (thought to reflect cholesterol deposits) seen in up to $36 \%$ of endometriomas. Endometriomas tend to be multilocular and bilateral (up to 50\%) [42] (Fig. 3).

However, endometriomas may have a variable appearance because of the range of appearance of the internal blood products within them, which can cause fluid-fluid

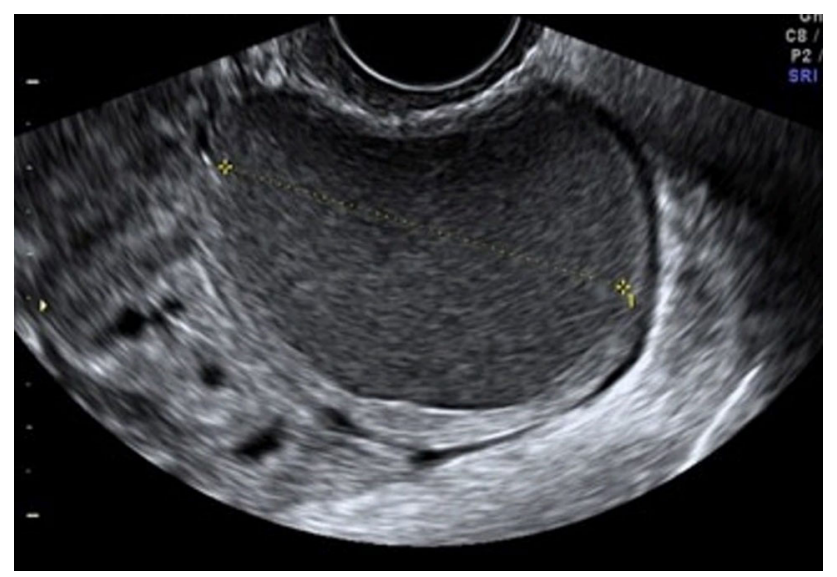

Fig. 1 Endometriotic cyst, with regular parenchyma at the periphery, called "crescent sign" characteristic of benign lesions. Absent intralesional vascularization levels, echogenic regions, and papillary projections. In these cases, additional evaluation with MR imaging may be warranted to better evaluate and to exclude malignancy [43]. There is evidence that ovarian endometriomas originate from ovulatory events and it is likely that the number of endometriomas may increase with age, and multiple endometriomas in the same ovary may assume a multilocular morphology [44]. Guerriero and colleagues reported that ultrasound appearance of endometriomas differed between premenopausal and postmenopausal patients [44]. The endometriomas in the postmenopausal patients were less often unilocular cysts and less likely to exhibit ground glass echogenicity [27].

The primary differential diagnosis of an endometrioma is a hemorrhagic cyst. On US, a hemorrhagic cyst classically has internal reticular strands with retractile clot [18]. However, these features may not be seen, and instead, homogeneous low-level echoes mimicking that of an endometrioma may be present. Hemorrhagic cysts are unlikely to have the peripheral echogenic foci occasionally seen in endometriomas, and they are less likely to be bilateral or multifocal. Sonographic follow-up demonstrating resolution at 6-12 weeks is diagnostic of a hemorrhagic cyst [18].

Another differential diagnosis of an endometrioma is an ovarian epithelial neoplasm, which may contain low-level internal homogeneous echoes similar to an endometrioma. This imaging appearance was seen in up to $6 \%$ of ovarian serous cystadenomas in the study by Patel et al. [42] and in up to $20 \%$ of mucinous cystadenomas in the study by Van Holsbeke [45]. To better evaluate for the presence of malignancy (cystadenocarcinomas) in these cases, careful interrogation of the cyst should be performed to assess for internal solid components, such as papillary projections, mural nodules, and thickened septations.

Doppler helps avoid classifying malignancies as endometriomas, especially when evaluating a papillary

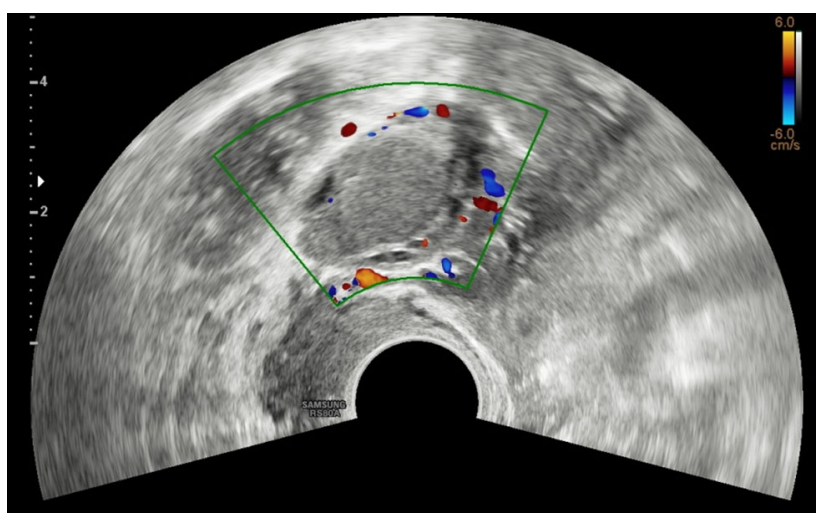

Fig. 2 Color Doppler of multiple endometriomas of both ovaries, which appear enlarged and sharpened (ovarian kissing) 


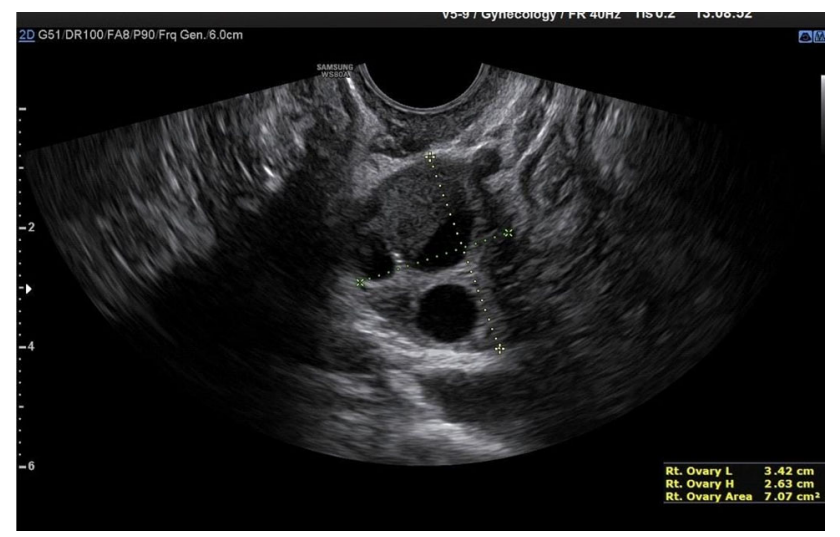

Fig. 3 Endometrioma with evidence of the characteristic double fluid-fluid level. Inside the formation, hyperechoic spots can be highlighted, a symptom of hemosiderin accumulation

projection. Generally, these different ultrasound criteria proposed have a sensitivity ranging from 62 to $73 \%$, a specificity of 94-98\% [46].

Masses in postmenopausal women whose cystic contents have a ground glass appearance have a high risk of malignancy. Borderline tumors and carcinomas arising from endometrioid cysts show a vascularized solid component on ultrasound examination [47].

\section{Tubes}

The uterine tubes can be involved with endometriosis either with adhesions occluding the tube up to $6 \%$ ) or by DIE foci affecting the tubal walls (up to $26 \%$ of the time) [18].

In case of endometriosis of the tube, we can observe a dilated tube with thick walls and incomplete septa with a fluid dense content similar to an endometrioma (hematosalpinx) [48]. A "cog-wheel" appearance of the longitudinal folds can be seen when the tube is imaged in cross-section. The presence of a hematosalpinx may be the only sign on imaging of endometriosis in the pelvis [49].

In case of occlusion of the tube due to adhesion or DIE that involved the distal part and the fimbriae, a hydrosalpinx is seen with the typical "beads-on-a-string" sign, defined as hyperechoic mural nodules measuring approximately $2-3 \mathrm{~mm}$ as seen on the cross-section of the fluidfilled distended structure [48].

The differential diagnosis of a hematosalpinx includes pelvic inflammatory disease (PID) or fallopian tube malignancies. Pyosalpinx of PID can be differentiated clinically by the presence of extreme tenderness on examination as well as the clinical signs of infection (fever, white count). On imaging, hyperemia surrounding the fallopian tube with fatty proliferation/edema in the adjacent fat suggests a pyosalpinx. Fallopian tube carcinoma presents sonographically with solid, vascular internal nodules within the fallopian tube and tends to occur in an older demographic group [43].

\section{Posterior Compartment}

Recently, the ultrasound features of the deep infiltrating endometriosis nodules have been systematically defined by the International Deep Endometriosis Analysis group [34]. The most common sites of the posterior compartment are posterior vaginal fornix/rectovaginal septum, uterosacral ligaments, anterior rectum/anterior rectosigmoid junction, and sigmoid colon [10] (Fig. 4).

Deep endometriosis on sonography is subtle and presents as hypoechoic nodular or infiltrating regions. Occasionally, the infiltrative regions of DIE may have internal hyperechoic foci or complex internal cysts [50]. The differential diagnosis for DIE includes peritoneal implants; in these cases, to help differential diagnosis, an additional evaluation with MR is recommended [18]. Three-dimensional (3D) TVS has been also proposed in the evaluation of posterior locations of DIE without intestinal involvement, improving the diagnostic accuracy of $2 \mathrm{D}$ ultrasonography [51].

In the cases of DIE, it is necessary to describe of the anatomical localizations, the size and number of DIE nodules, the depth of infiltration of the nodules, and the degree of stenosis of the bowel lumen which is important to plan the surgical procedures [22].

\section{Uterosacral Ligaments}

The uterosacral ligaments (USL) are usually not visible on ultrasound. The uterosacral ligaments affected by deep

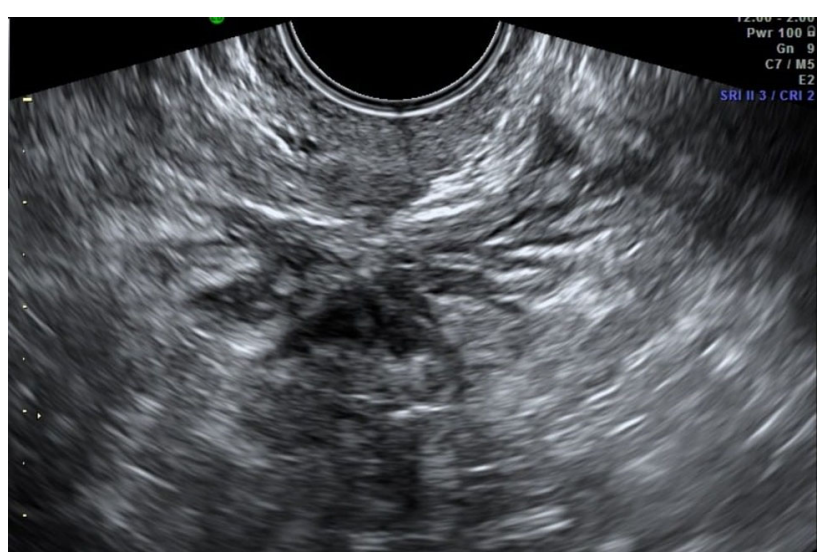

Fig. 4 Hypoechoic nodule of the rectosigmoid portion that obliterates the Douglas. At this level, the "sliding sign" can be seen, the sign of the sliding structures on each other which does not occur in the case of endometriosis 
infiltrating endometriosis can be seen in the longitudinal view of the uterus at the insertion on the posterior lateral cervix wall, as hypoechoic tissue, with regular/irregular margins within the peritoneal fat surrounding the uterosacral ligaments [13]. On the transverse cervical section, these hypoechoic nodules appear on the posterior lateral part of the cervix and interrupt the hyperechoic external cervical fascia. Sometimes, the uterosacral ligaments appear thickened and hyperechoic, probably as the morphologic expression of fibrosis, due to the chronic process of inflammation [27].

USLs lesions may be isolated or may be part of a larger nodule extending into the vagina or into other surrounding structures. In some cases, the DIE lesion involving the USL is located at the torus uterinus as a central thickening of the retrocervical area between USLs [52]. Two recent metaanalyses of USL endometriosis have reported pooled sensitivities and specificities of 53-64\% and 93-97\%, respectively [53].

In case of endometriotic lesions involving the uterosacral ligaments, special attention must be paid to the parametrium. Parametria are examined lateral to the uterine cervix first on the sagittal planes moving the probe from the lateral sites where the parametrium is attached to the cervix, to the uterine vessels bifurcation, to the lateral pelvic wall, and then on the transverse planes moving the probe from the uterine isthmus to the external cervical os. The parametrial involvement is seen as an infiltrating hypoechogenic irregular tissue, and it can be medially delimitated from the cervical vascular plexuses using color or power Doppler [22].

\section{Rectovaginal Septum}

Involvement of the rectovaginal septum should be suspected when an endometriotic nodule, which appears as hypoechoic solid nodule with smooth or irregular contours, that replaces the normal hyperechoic aspect of this layer between the vagina and the rectum, seen in the rectovaginal space below the horizontal plane passing through the lower border of the posterior lip of the cervix (under the peritoneum) [34].

Isolated rectovaginal septum nodule is rare, and it is usually an extension of posterior vaginal wall, anterior rectal wall, or both posterior vaginal wall and anterior rectal wall involvement. Hourglass-shaped or diabolo-like nodules can occur when endometriosis lesions from the posterior vaginal fornix extend to the anterior rectal wall [54].

DIE of the RVS may extend into the rectum and/or in the posterior vaginal fornix [22].

Major discrepancies exist between the pooled sensitivities and specificities provided by meta-analyses reporting values from $49 \%$ to $88 \%$ and $98 \%$ to $100 \%$, respectively [34].

\section{Vagina}

Vaginal endometriosis is diagnosed when the posterior or a lateral vaginal fornix shows a nodular wall thickened (> $5 \mathrm{~mm}$ ) (mean normal vaginal thickness ranges from 3 to $5 \mathrm{~mm}$ ), with or without round cystic anechoic areas, that does not get thinner with probe compression [13].

The nodule may be hypoechoic, homogeneous, or inhomogeneous with or without cystic areas and there may also be some vascularization at power Doppler. More frequently, the lesions are localized in the posterior vaginal fornix [22].

The insertion of saline solution in the vagina (sonovaginography) could improve the visualization of these lesions [55].

In a meta-analysis including ten studies, the pooled sensitivity and specificity of TVS was $57 \%$ and $99 \%$, respectively [56]. Among the various TVS techniques, SVG provided the highest sensitivity and specificity reaching $91 \%$ and $89 \%$, respectively [56].

\section{Bowel}

The endometriosis affecting the bowel can appear as a thickening of the muscularis propria or as a hypoechoic nodule penetrating the intestinal wall with blurred margins, with or without hypoechoic or hyperechoic foci, usually associated with retraction and adhesion (the so-called Indian headdress sign), and few vessels at power Doppler evaluation [57].

The rectum and the rectosigmoid segment is the most frequent site of bowel involvement accounting for 70-88\% of cases of bowel involvement with endometriosis, followed by the sigmoid colon, rectum, ileum, appendix, and cecum [10]. Intestinal nodules located below the peritoneum of the POD (or the level of the insertion of the USLs on the cervix in case the cul-de-sac is obliterated) are considered low rectal lesions, while the ones above this level are considered upper rectal or the rectosigmoid junction lesions. This virtual line should delineate the plane under the peritoneum of the POD and correspond laterally to the parametria and medially to the RVS. The lowest limit of the nodule on the bowel wall should be determined, because the lower rectal lesions are more difficult to remove surgically by shaving or segmental resection and have higher complication rate [22].

Endometriotic nodules of the rectum can be evaluated if necessary also by transrectal examination as well with the same transvaginal convex probe. This has the advantage of visualizing better the vagina, the rectovaginal septum 
(RVS), and the low rectal walls. Moreover, during the transrectal or transvaginal examination, a fluid contrast medium can be inserted in the vagina to visualize better the RVS (sonovaginography) [55]. It has been reported that adding water contrast in the rectum during transvaginal ultrasonography (RWC-TVS) improves the diagnosis of rectal infiltration in women with rectovaginal endometriosis. RWC-TVS is performed by injecting saline solution into the rectal lumen under ultrasonographic control through a catheter [58].

During the evaluation of posterior compartment, a negative "sliding sign" between the rectosigmoid and uterus could indicate an obliteration of the pouch of Douglas (POD), frequently associated with severe DE. Using this new technique, Reid et al. found sensitivity, specificity of $83.3 \%, 97.1 \%$ [59].

Multifocal lesions are defined as the presence of deep lesions within 2-cm area of the main lesions or multiple endometriotic lesions affecting the same intestinal segment. Multicentric lesions are defined as a satellite deep nodule found more than $2 \mathrm{~cm}$ from the main lesions or endometriotic lesions affecting several digestive segments [60].

The use of volume acquisition with 3D TVS permits a more accurate measurement and evaluation of the DIE lesion in different planes.

The pooled sensitivity and specificity of TVS for rectosigmoid endometriosis are reported as $90 \%$ and $96 \%$, respectively, with similar results being provided by RES [56]. Guerriero et al., in a one-paired study, suggested that 2D-TVS was more sensitive but less specific than 3D TVS [61].

\section{MRI}

\section{Protocols}

MR imaging of the pelvis is frequently performed for the detection of endometriosis, either as the second-line imaging examination (after US) for the detection/confirmation of endometriosis, in particular in deep infiltrating endometriosis, or as the initial examination in a patient for whom there is a high clinical suspicion for endometriosis and not ultrasound confirm [1].

MR imaging can be performed with either a 1.5-T or 3-T magnet, using a high-resolution phased-array surface coil for improved resolution. There is no consensus regarding whether to perform the examination around the timing of the patient's menstrual cycle [13].

Patients are positioned supine on the scanner, with abdominal strapping after phased coil array placement. Fasting before the examination for $4 \mathrm{~h}$ is typically recommended in order to empty the upper gastrointestinal tract; the use of antiperistaltic agents is recommended to reduce motion artifacts caused by intestinal peristalsis. However, the type of agent (oral agents, nonoral agents), dose, and route (intramuscular, subcutaneous, or IV) is debated [18].

Patient preparation required a moderately filled bladder; this is required to change the angle of uterine anteversion, leading to better detection of implants in the anterior compartment. Moreover, a moderately filled bladder displaces the bowel superiorly, by reducing the artifacts from bowel motion [62].

For the evaluation of deep endometriosis it was suggested to introduce intra-vaginal aqueous gel to distend the vaginal cavity and better explore the vaginal fornices and the retrocervical area [62].

In the presence of symptoms that may be related to rectal involvement, gel may also be useful to distend the rectallsigmoid bowel wall.

The typical imaging protocol $[21,63]$ includes three T2 turbo-spin-echo (TSE)—weighted sequences (T2W) in different slice orientations (sagittal, coronal, and axial planes), followed by three T1-weighted (T1W) sequences in an identical imaging plane (TR $500 \mathrm{~ms}$, TE $14 \mathrm{~ms}$ ) without fat suppression and fat-suppressed $\mathrm{T} 1 \mathrm{~W}$ before and after intravenous injection of contrast media because the fat suppression is useful for the detection of subtle foci of hemorrhage, which may be obscured on non-fat-saturated images [64].

Use of contrast-enhanced imaging is primarily required to identify solid enhancing nodules within endometriotic cysts when malignant transformation is suspected.

Dixon technique or conventional in- and out-of-phase T1-weighted images are useful for the differentiation of fat-containing lesions, such as dermoid cysts from endometriomas, both of which have high signal on non-fatsaturated T1-weighted images [18].

No recommendation can be achieved for the use of DWI and SWI sequences.

Half-Fourier acquisition single shot turbo-spin-echo (HASTE) is recommended for the evaluation of uterine peristalsis because it enables multiphase and multislice image acquisition producing kinematic images for the evaluation of pelvic adhesions [1]. During the peri-ovulatory phase, uterine peristalsis is significantly reduced in subjects with endometriosis when compared to normal controls that may be due to increased, sustained contractions in endometriosis patients [65].

\section{MRI Evaluation}

At MR imaging the signal intensity of endometriotic lesions is a function of the quantity and age of the hemorrhage on the one hand and the proportion of endometrial cells and stroma on the other [66]. 
The lesions have a micronodular or microcystic appearance; however, cysts do not enlarge except in the ovary. Only pigmented lesions can be detected at noncontrast-enhanced MR imaging because of the presence of hemorrhage [67]. At MR imaging these small implants manifest as multiple round (cystic or nodular) lesions homogeneously hyperintense on fat-suppressed T1-weighted images, due to old hemorrhagic content, regardless of their signal intensity on T2-weighted images [67]. Involvement of peritoneal reflections over the cul-de-sac and the uterus may also manifest on contrast-enhanced fatsaturated T1-weighted images as diffuse peritoneal enhancement secondary to the inflammatory reaction induced by endometrial implants. Over time a fibrotic reaction may occur, thus leading to adhesions formation between pelvic structures [67].

T2W sequences are used for the evaluation of fibrotic lesions, notably those that involve the pelvic ligaments, retrocervical space, or prevesical recess [21].

They appear as speculated hypointense peritoneal strands arranged in confluent angles. Posterior displacement of uterus and ovaries, angulation of rectosigmoid colon and bowel loops, elevation of the posterior vaginal fornix, loculated fluid collections, and a hydrosalpinx may be indirect signs of adhesions [68].

At MR imaging both fibrous tissue and smooth muscle show intermediate signal intensity on T1-weighted images and low-signal intensity on T2-weighted images. Therefore, on T2-weighted images, solid endometriotic lesions appear as hypointense nodular structures with irregular or stellate margins due to fibrous tissue and smooth muscle proliferation. In certain cases, deep endometriotic lesions may also appear as irregular and hypointense soft-tissue nodular thickening on T2-weighted sequences, as it occurs when the disease involves the USL or the vaginal or rectal wall [66].

In some cases the glandular component can be predominant, compared to the fibrous tissue and in this case the MRI appearance will show high signal intensity of T2W images; in this case the use of contrast material can be useful because this will show enhancement, thus distinguishing it from intramural hemorrhage or necrosis [63]. Usually, endometrial glands without hemorrhage are not detectable on fat-suppressed T1-weighted images; so deep lesions may show homogeneous intermediate signal intensity on T1-weighted images. When red cell extravasation outside the glandular ducts into the surrounding stroma occurs, these small hemorrhages become visible as small hyperintense spots on fat-saturated T1-weighted images. After the intravenous administration of gadolinium, lesion enhancement may occur due to inflammatory reaction, glandular and fibrous tissue.
Owing to the possibility to perform a complete assessment of all pelvic compartments at one time, MRI represents the best imaging technique for preoperative staging of endometriosis [66].

\section{Anterior Compartment}

Endometriotic lesions may affect the urinary tract in up to $20 \%$ of cases.

\section{Bladder}

Bladder lesions appear as small masses of round or lobulated hypertrophic tissue covered by normal mucosa [67].

Bladder involvement is often multifocal, the trigone and the dome being the most frequently affected sites [23]. According to the degree of wall infiltration, bladder involvement may be classified as extrinsic or intrinsic. In extrinsic involvement, the most common form, implants are confined to the serosal surface or the surrounding connective tissue; in intrinsic involvement lesions infiltrate the muscular layer manifesting as mural masses [66].

At MR imaging bladder endometriosis may manifest as localized or diffuse wall thickening and signal intensity abnormalities [63]. The appearance is of low-signal intensity on $\mathrm{T} 2$-weighted and intermediate signal intensity on T1-weighted images, with or without spots of high signal intensity on T1-weighted images, representing hemorrhagic content. Sometimes, hyperintense foci on T2W images corresponding to the dilated endometrial glands may be found [66]. Implants minimally enhance after injection of a gadolinium-based contrast material [64].

The maximum lesion diameter varies between 1 and $5 \mathrm{~cm}$. MRI reaches sensitivity up to $88 \%$, specificity up to $99 \%$ and diagnostic accuracy of about $98 \%$ for the diagnosis of bladder endometriosis [23]. The differential diagnosis of bladder endometriosis includes urachal remnant, epithelial tumors (bladder carcinoma) and mesenchymal tumors (angiomas, leiomyoma) [23].

\section{Urethra}

Urethral endometriosis is uncommon; direct implantation of endometrial tissues during procedures is hypothesized to be the etiologic mechanism [69]. In some cases, endometriosis may be mistaken for a urethral diverticulum and therefore a precise diagnosis is essential to facilitate optimal management. Usually, urethral involvement is observed as a contiguous extension from the bladder and the MRI findings are the same as those described for bladder endometriosis [64]. 


\section{Ureter}

Ureteral endometriosis may be defined as any situation where endometriosis or surrounding associated fibrosis causes compression or distortion of the normal ureteral anatomy, even when hydroureteronephrosis is not yet present [70]. Ureteral endometriosis is quite uncommon, most often unilateral, with a left predisposition; bilateral involvement is present in approximately $10-20 \%$ of cases [71]. Ureteral involvement is often associated with an ipsilateral endometrioma or with a rectovaginal nodule larger than $3 \mathrm{~cm}$. The distal ureter, $3-4 \mathrm{~cm}$ above the vesicoureteral junction, is the most common ureteral segment involved [71].

The symptoms can vary according to the type of nodule infiltration: indeed endometrial tissue can directly infiltrate the muscularis propria, lamina propria, or ureteral lumen causing symptoms that may be related to the pelvic endometriosis itself (dysmenorrhea, dyspareunia) or secondary to urinary tract involvement (flank pain, obstruction, and in some cases decline of renal function) [64]. Also in the ureteral endometriosis there are two major pathological types: extrinsic and intrinsic. Extrinsic endometriosis is the most common form (80\%) of ureteral involvement and it represents endometrial glandular and stromal tissue within the submucosa and adventitia of the ureter [21]. In addition, scar tissue or fibrosis without true endometriotic invasion of the ureter may also be classified as extrinsic disease. In contrast, intrinsic endometriosis (about 20\%) involves the uroepithelial and muscular layer. At MRI, ureteral endometriosis usually appears as hypointense solid nodules on $\mathrm{T} 2 \mathrm{~W}$ images with spiculated margins, that envelop the ureter, causing dilatation of the ureter upstream [72]. Extrinsic disease may be hypothesized when the interface of fat between the nodule and ureter is no longer visible. MR urographic techniques can be used to obtain three-dimensional reconstructed images from coronal volumetric excretory phase $\mathrm{T} 1 \mathrm{~W}$ data. The differential diagnosis of ureteral endometriosis includes ureteral invasion by cervical cancer [64].

\section{Vesicouterine Pouch}

The vesicouterine pouch or anterior cul-de-sac is a common site of endometriotic involvement [21]. These lesions are associated with anteflexion of the uterus and obliteration of the anterior cul-de-sac due to extensive adhesions between the peritoneum of the bladder fold and the uterus. At MR imaging deep endometriotic implants involving the anterior uterine serosa demonstrate infiltrative pattern with indistinct margins and show hypointense nodules on T2weighted images, with small cystic areas that typically adhere to the anterior uterine surface, forming an obtuse angle with the vesical wall [72].

\section{Central Compartment}

\section{Ovaries}

The ovaries are the most common site of endometriosis (20-40\% of cases) [72]. They may be affected in two ways: (i) the endometriomas or chocolate cysts that are associated with ovarian enlargement, and which are caused by repeated episodes of hemorrhage; and (ii) small nodular superficial implants which may cause paraovarian fibrous scarring and adhesions [64] (Fig. 5A-C).

Peritoneal implants confined to the ovarian surface are often underdiagnosed at imaging due to their small size $(<5 \mathrm{~mm})$ [66].

Endometriomas are frequently multilocular and bilateral (50\% of cases). In MRI an endometrioma appears as a homogeneously hyperintense mass on T1W MR images; on T2W MR images, it appears as a low-signal intensity mass with areas of high signal intensity [67] (Fig. 6A-F). Endometriomas have a wall with a variable appearance (from thin to thick and fibrotic) and they usually contain dark/brown semi-solid material that represents the degenerated blood products (the so-called "chocolate cyst") [67] (Fig. 7A-E). Because the endometriomas contain blood products of different ages and concentrations, they may show a variable appearance, in fact as free water in the cyst is resorbed, the iron concentration increases along with the viscosity of the contents of the cyst: this condition determines the "shading effect". Shading is present when a cyst, hyperintense on a T1W image, shows a gradient from hypointense to hyperintense on a T2W image. Shading can range from faint, dependent layering to complete signal void, according to the concentration of blood products [64]. It reflects the chronic nature of endometriomas and is the result of cyclic bleeding occurring over time. Old blood products contain high iron and protein concentrations which determine a decrease in $\mathrm{T} 2$ relaxation time. Therefore, on T2-weighted images endometriomas will show a gradual loss of signal within the lesion with low-signal intensity till complete signal void in the declivous portion.

The most specific pathologic feature of endometrioma is the thick fibrous capsule containing a cluster of hemosiderin-laden macrophages due to repeated hemorrhage [66].

In certain cases, the ovaries may be joined together behind the uterus in the pouch of Douglas due to adhesion formation between the adjacent peritoneal surfaces, a sign described at US as B kissing ovaries ${ }^{\wedge}$ and suggestive of severe pelvic endometriosis [73]. 
a
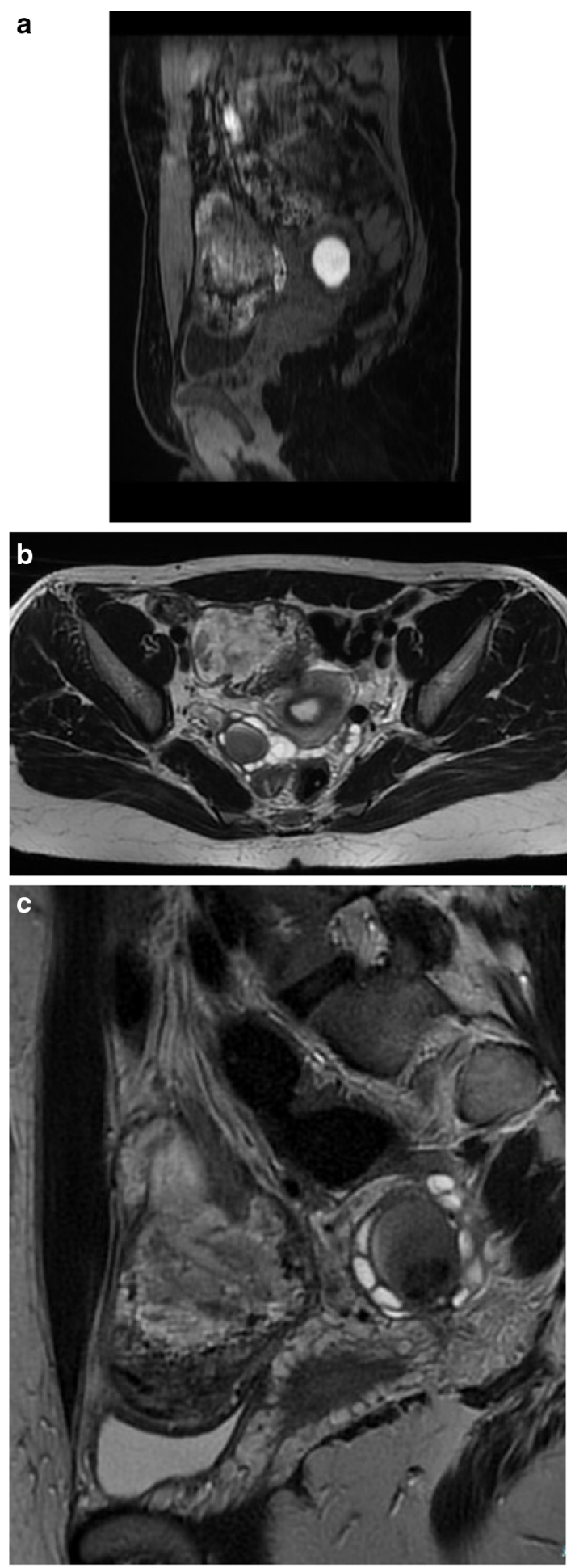

Fig. 5 An Example of multiple endometriomas of both ovaries, which appear enlarged and sharpened (ovarian kissing): A sagittal T1w fat sat imaging; B, C axial and sagittal T2w imaging

Togashi et al. found that an extremely sensitive sign for the presence of an endometrioma was the presence of a cyst hyperintense on T1W images and the presence of shading on $\mathrm{T} 2 \mathrm{~W}$ images [74]. Another criterion is to observe multiple hyperintense cysts on T1W images (and T1W fatsuppressed images) regardless of their signal intensity on T2W images (Fig. 8A-F).

The differential diagnosis of endometriomas includes lesions with high signal intensity on T1-weighted images: dermoids, mucinous cystic neoplasms, and hemorrhagic masses. Fat-saturated T1-weighted sequences are helpful to rule out a fat-containing lesion (such as dermoids) and to confirm the presence of blood [75]. Mucinous lesions may show hyperintensity on T1-weighted images, but signal intensity is lower than that of blood.

It is possible to recognize and differentiate dermoids from endometriomas by the presence of chemical shift artifact and signal drop-out on the fat suppression image. Thus, on a T1-weighted frequency selective fat saturation sequence, the mature cystic teratoma will be low in signal, whereas an endometrioma will have high signal [64].

Hence, the most challenging differential diagnosis is with other hemorrhagic masses. To differentiate endometriomas from functional hemorrhagic cysts is important in order to prevent unnecessary surgical interventions. Functional hemorrhagic cysts (i.e., hemorrhagic follicular cysts and hemorrhagic corpus luteum cysts) are usually unilocular and unilateral, do not display shading on T2-weighted images, and mostly disappear on follow-up examinations (generally in 4-6 weeks), while endometriomas are usually multilocular and bilateral [68].

The role of DWI sequences in differentiating endometriomas from functional hemorrhagic ovarian cysts is still debated. Balaban et al. found significantly lower ADC values in endometriomas compared with functional hemorrhagic ovarian cysts in all b values [76]. Large lesions with wall nodularity, thick septations and enhancing solid components may be suggestive of malignancy.

\section{Fallopian Tubes}

Endometriotic involvement of the fallopian tubes is strongly associated with infertility. Serosal or subserosal implants involves the peritoneal surface of the fallopian tubes, where repeated hemorrhages lead to fibrosis and retraction of the tube with hydrosalpinx. Intraluminal implants determine cyclic hemorrhage thus causing hematosalpinx [66].

Moreover, an association has been described between the endometriosis in the fallopian tubes and a predisposition to endometrial malignancies such as clear cell carcinoma and endometrioid carcinoma [67].

At MR imaging hematosalpinx appears as a tortuous enlarged tubular adnexal structure filled with hemorrhagic fluid. Endoluminal content shows high signal intensity on fat- suppressed T1-weighted images and intermediate signal intensity, (40\% of distended tubes in endometriosis 

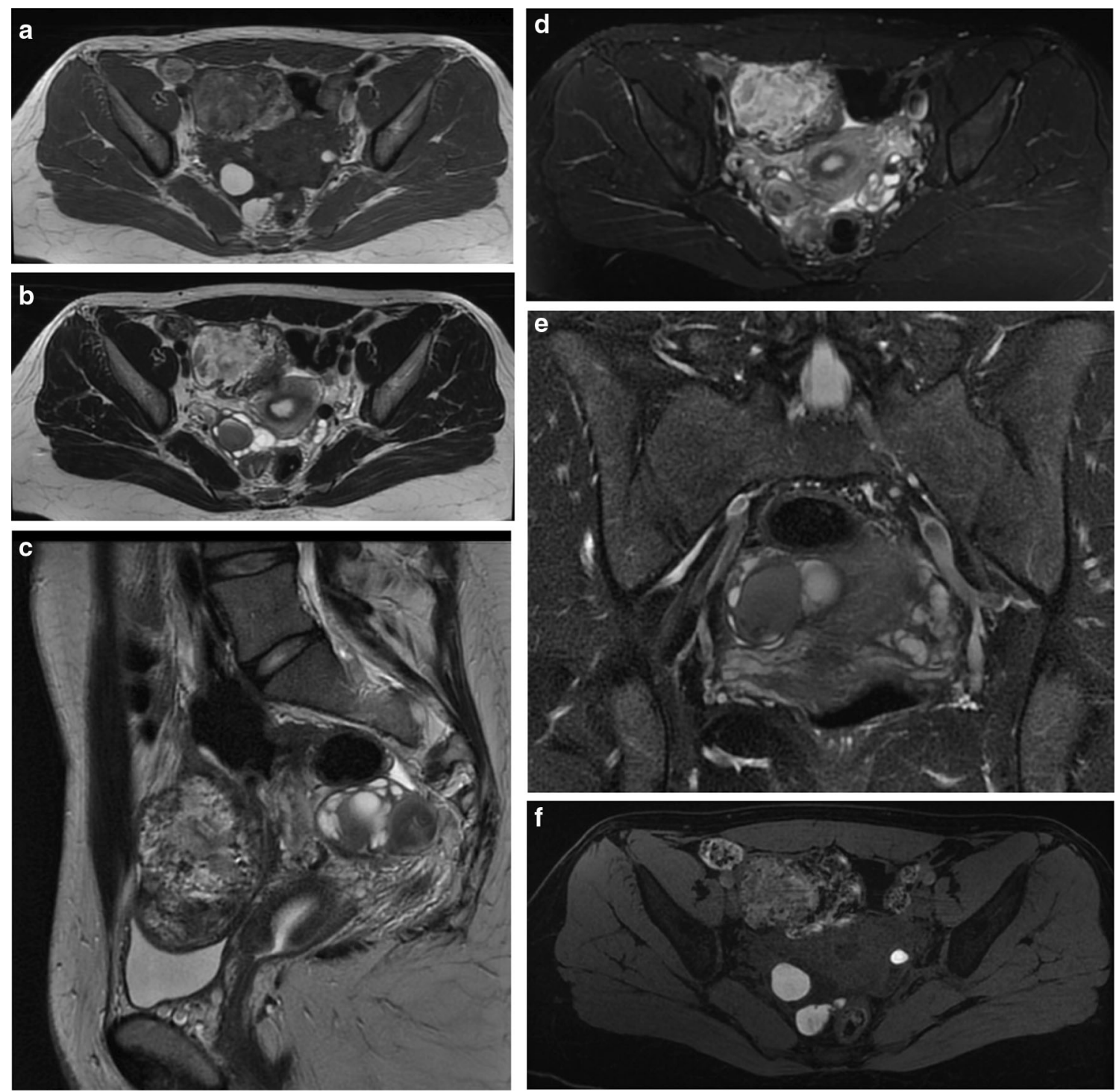

Fig. 6 An example of ovarian endometriosis: A T1w imaging; B, C axial and sagittal T2w imaging; D, E axial and coronal T2 fat sat imaging; F T1w imaging after contrast administration. Hyperintensity in T1w is typical of a recent bleeding

have hyperintense contents) with or without internal fluidfluid level, on T2-weighted images [77]. According to Siegelman the presence of T1-weighted hyperintensity within a dilated fallopian tube is suggestive of endometriosis [78].

In addition, it is atypical to see $\mathrm{T} 2$ shading within the lumen of the distended fallopian tube even when there is high signal on T1-weighted images [78]. T2 shading is not seen because of the fact that the endometriotic implants are mostly along the surface of the tube and not within the lumen of the tube, such that the chronic bleeding within the implants leads to adhesions along the tubal surface, but not within the lumen. The differential diagnosis of a hematosalpinx on MR imaging includes PID or fallopian tube malignancies. Pyosalpinx of PID can be differentiated by the clinical signs of infection (fever, white count) [18].
On MR imaging, hyperemia surrounding the fallopian tube with stranding in the adjacent fat would suggest a pyosalpinx. Fallopian tube carcinoma demonstrates solid, enhancing internal nodules within the fallopian tube and tends to occur in an older demographic group [43].

\section{Uterus and Vagina}

The most common localization of ectopic endometrial tissue within the uterus is the adenomyosis. Whereas it is important to remember that uterine involvement by endometriosis is usually subserosal, sometimes it is possible to find nodules of endometriosis in the serosal surface of the uterus [64].

Vaginal endometriosis is usually associated with implants in other pelvic locations, mostly retrocervical and 

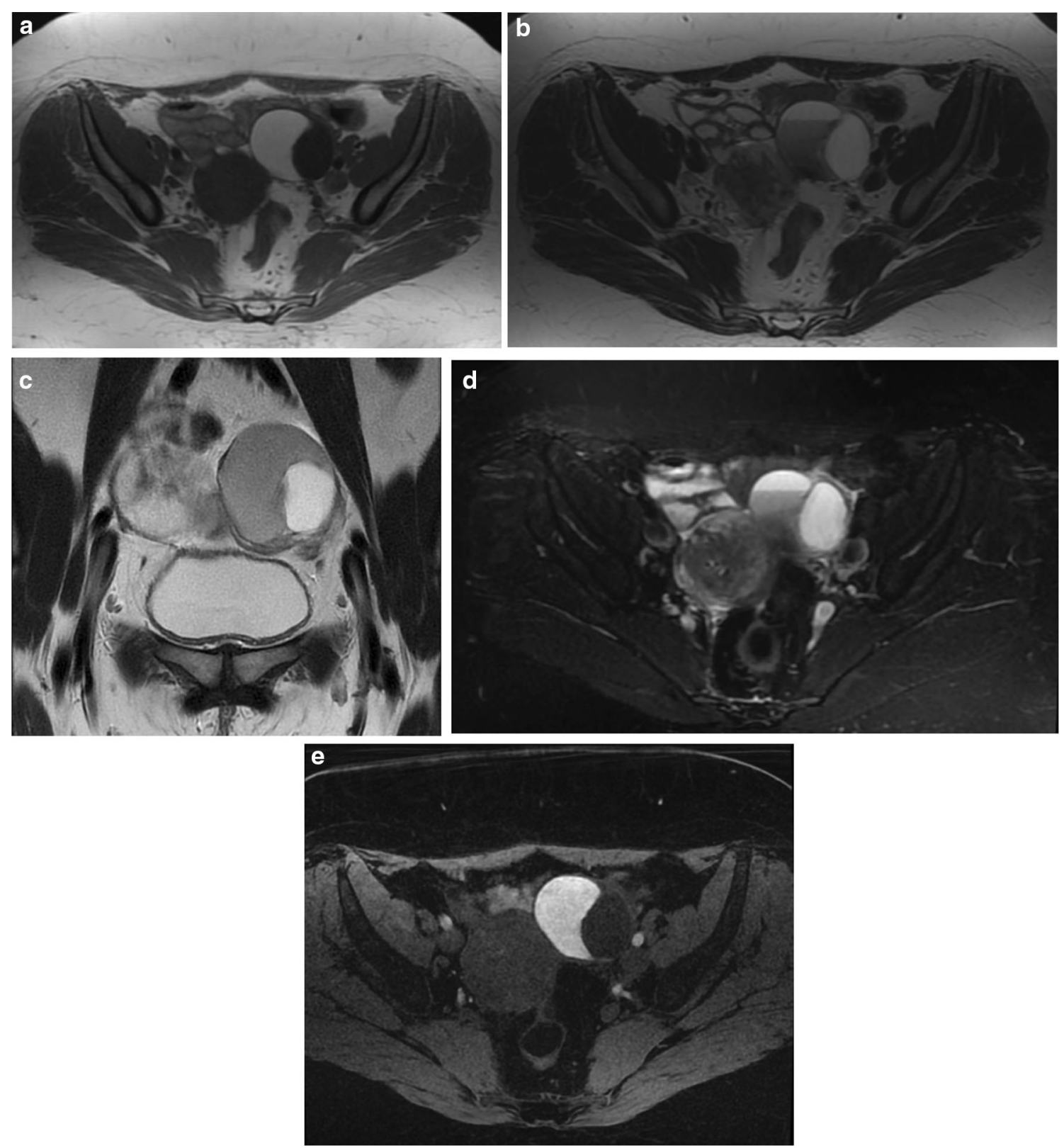

Fig. 7 An example of endometrioma: A T1w imaging; B, C axial and coronal T2w imaging; D axial T2 fat sat imaging; E axial T1w imaging after contrast administration. It's visible a double fluid-fluid level, indicating different bleedings

rectal lesions; seldom isolated involvement of the vagina may occur. The upper one-third of the vagina and the posterior fornix are the most commonly affected sites. Generally, the vaginal wall implants show a thickened or nodular appearance [21], but may also have a polypoid structure. At MR imaging vaginal endometriotic implants show low-signal intensity on T2-weighted images. They often have a multiloculated internal appearance because of the presence of cystic areas [66]. These locules can show hyperintense content on T1-weighted images due to subacute blood products. Polypoid variant may have a T2 hypointense rim corresponding to surrounding fibrous tissue associated with endometriosis [79]. Rectovaginal fistulation represents a complication of vaginal endometriosis. Differential diagnosis includes epithelial neoplasms arising from the uterine cervix or vaginal wall [66].

\section{Uterine Ligaments}

At MRI the round ligaments can be identified as thin structures with hypointense fibrous signal on T1- and T2weighted images, extending from the uterine horns to the pelvic side- wall, passing anteriorly to the external iliac 

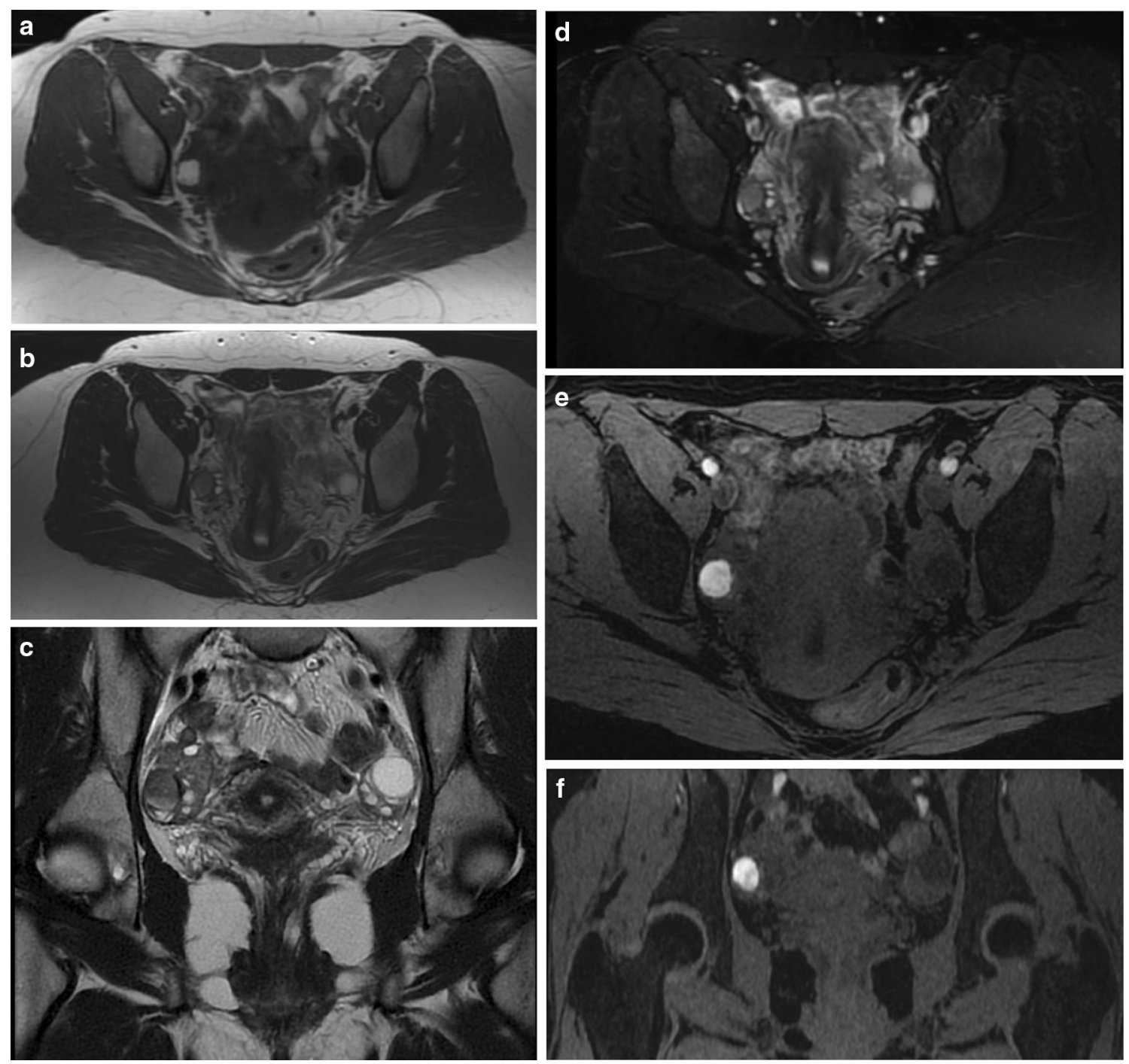

Fig. 8 An example of endometriotic cyst: A axial T1w imaging; B, C axial and coronal T2w imaging; D axial T2 fat sat imaging; E, F axial and coronal $\mathrm{T} 1 \mathrm{w}$ imaging after contrast administration

vessels. They have an intra- and an extra-pelvic portion, the latter being the distal part of the ligament in the canal of Nuck [80]. When involved by endometriosis, round ligaments appear thickened (more than $1 \mathrm{~cm}$ ), nodular, shortened and irregular. Usually endometriotic implants are a mixture of fibrous tissue and hemorrhage. Fibrous tissue shows hypointense signal on T1- and T2-weighted images; small hemorrhagic foci displays hyperintense signal on fatsuppressed T1-weighted images [66]. The presence of free fluid around the intra-pelvic portion of the round ligaments may represent an indirect sign of endometriosis [80].

Endometriosis of the broad ligaments usually manifests as thickening and nodularity of these peritoneal folds extending between the uterus and the lateral walls of the pelvis. These nodules are visible as hypo-intensity signal in $\mathrm{T} 2$ sequences and after administration of contrast material it is possible to observe a diffuse peritoneal enhancement secondary to the inflammatory reaction incited by microscopic endometrial implants on contrast-enhanced fat-saturated MRI [64].

\section{Posterior Compartment}

\section{Uterosacral Ligaments}

Uterosacral ligaments (USL) are the most frequent location of deep endometriosis. Bilateral USL involvement is often associated with other posterior deep endometriotic locations, mostly the rectosigmoid colon [81]. At MR imaging normal USL are depicted as thin, regular, semicircular hypointense cords that originate from the lateral aspect of the uterine cervix and the vaginal vault and course dorsocranially toward the sacrum [21]. USL endometriosis is 
depicted as nodularity within the ligament or as unilateral or bilateral hypointense thickening of the ligament, with regular or irregular margins [21]. The proximal medial portion of the USL is most commonly affected by endometriosis.

According to Bazot et al. thin-section oblique axial T2weighted sequences ( $3 \mathrm{~mm}$ thick, perpendicular to the long axis of the cervix) can improve the capability of conventional MRI to assess USL endometriosis [81].

Saba et al. suggested that the diagnosis of endometriosis of the USL is simple when ligaments are involved together with the torus uterinus, whereas, when there is only a thickening or an asymmetric nodular irregularity the involvement of the USL can be difficult [64].

In a recently published meta-analysis the sensitivity and specificity of MRI for the diagnosis of endometriosis of USL were $85 \%$ and $80 \%$, respectively [82].

\section{Retrocervical Area}

The retrocervical area is a virtual extraperitoneal space behind the cervix, located above the rectovaginal septum [21]. It is a common site of deep pelvic endometriosis. Retrocervical implants are often associated with USL involvement and with the retroversion of the uterus [83].

Deep endometriotic lesions of the retrocervical area frequently appear as ill-defined infiltrative tissue, hypointense on $\mathrm{T} 2$-weighted images, extending from the posterior uterine serosa to the retrocervical region [21]. Nevertheless, some lesions may contain abundant glandular component and little fibrotic reaction, thus showing high signal intensity on $\mathrm{T} 1$-weighted images and variable signal intensity on T2-weighted images [21, 63]. The solid glandular component enhances after intravenous administration of contrast material [63].

Del Frate et al. identified a condition they called "hourglass-shaped" lesions that are found in $25 \%$ of cases and are due to posterior extension of a posterior forniceal lesion toward the anterior rectal muscularis. These lesions are usually larger than $3 \mathrm{~cm}$, with a greater risk of extension to the rectal wall [63].

In a recently published meta-analysis the sensitivity and specificity of MRI for the diagnosis of endometriosis of the pouch of Douglas were $89 \%$ and $94 \%$, respectively [82].

The differential diagnosis of retrocervical lesions includes peritoneal metastases from intraperitoneal malignancies (i.e., gastrointestinal and ovarian neoplasms). Peritoneal metastases usually show intermediate to high signal intensity on T2-weighted images and, as the primary cancer site, high signal intensity on DWI; moreover, ascites and a tumor mass into the abdominal cavity may be identified [84]. On the other hand, solid endometriosis shows low-signal intensity on T2-weighted images [66].

\section{Rectovaginal Pouch}

The rectovaginal pouch is the anatomical region located between the posterior vaginal wall and the anterior rectal wall. It extends from the deepest part of the pouch of Douglas to the top of the perineal body [66]. The inferior two thirds of this space constitute the rectovaginal septum, a thin membranous partition usually filled with fat [21]. Usually rectovaginal implants represent extensions from retrocervical or posterior vaginal lesions.

In MRI, nodules of endometriosis affecting the rectovaginal pouch usually appear as hypointense nodules on both T1W and T2W MRI images with signal intensity close to that of pelvic muscle [85]. Sometimes, foci of endometriosis may also have an abundant glandular component and discrete fibrotic reaction. In such cases, the endometriotic foci are hyperintense nodules on $\mathrm{T} 1 \mathrm{~W}$ and fat-saturated T1W MRI images, irrespective of their appearance on T2W MRI images. Moreover, the solid glandular component shows variable enhancement after the intravenous administration of contrast material [64].

In normal conditions the MRI depicts the rectovaginal septum as a hyperintense signal area in $\mathrm{T} 1 \mathrm{~W}$ and $\mathrm{T} 2 \mathrm{~W}$ images whereas nodules of endometriosis affecting the rectovaginal septum usually appear as hypointense nodules on both T1W and T2W MRI images [64].

In a recently published meta-analysis the sensitivity and specificity of MRI for the diagnosis of rectovaginal septum endometriosis were $82 \%$ and $77 \%$, respectively [82].

\section{Rectosigmoid Colon}

Among the bowel segments the rectosigmoid is the most commonly involved by endometriosis $(65.7 \%)$, followed by vermiform appendix, terminal ileum, cecum and descending colon, in order of frequency [86]. Rectosigmoid endometriosis is often associated with other pelvic locations and with a second intestinal lesion in $55 \%$ of cases [72].

The rectosigmoid endometriosis may cause adhesions, bowel strictures, or intestinal obstruction may result from the inflammatory response to cyclic hemorrhage. The implants are usually serosal but can sometimes involve the subserosal layers and cause thickening and fibrosis of the muscularis propria. Usually, an intact overlying mucosa is present, since the implanted tissue only rarely invades through to the mucosa [21].

Typically, endometriotic lesions infiltrating the anterior rectal wall have a characteristic fan-shaped configuration (or a pyramidal shape, with the base adhering to the rectal wall and the apex oriented anteriorly toward the retrocervical region). The core of the lesion shows isointense signal compared to muscle on T2-weighted and T1-weighted 
sequences and at histopathology corresponds to thickening and distortion of the muscularis propria and smooth muscle hyperplasia [66]. The overlying layer, hyperintense on T2weighted images, at the luminal side of the bowel wall corresponds to (sub)mucosal thickening, as a consequence of non-specific inflammation with or without infiltration of endometriosis [86]. When the longitudinal extent of the parietal lesion along the bowel wall is short, a pattern of intraluminal endophytic growth, called mushroom cap, may be observed [87].

When nodules of endometriosis are localized in the retroperitoneal section frequent concomitant findings are the adherences that identify fibrotic tissue originating from the nodules of endometriosis and involves the closest organs. Sometimes the adherences determine traction of the affected organs [88]. This is an important finding because it can be considered an indirect sign of endometriosis.

In a recently published meta-analysis the sensitivity and specificity of MRI for the diagnosis of rectosigmoid colon endometriosis were $83 \%$ and $88 \%$, respectively [82]. MR imaging is useful to predict infiltration of the muscular layer of the bowel with a sensitivity of $100 \%$ and specificity of $75 \%$. On the other hand, it is of limited value in diagnosing (sub)mucosal infiltration, as (sub)mucosal thickening may be caused by edema without infiltration of endometriosis. Nevertheless, extensive irregularities of the (sub)mucosal layer may raise suspicion of (sub)mucosal involvement [86]. Differential diagnosis includes rectal cancer and metastatic implants to the bowel [66].

\section{Conclusions}

Endometriosis is a chronic condition that affects women during the reproductive lifespan. Diagnosis of endometriosis is complex as it must take into account nonpathognomonic clinical symptoms and non-specific laboratory tests. The physical examination and above all the different imaging techniques, in particular US and MRI, constitute the gold standard for diagnosis. The role of the radiologist is fundamental both in the diagnosis of endometriosis, especially in the deep sites that cannot be seen with ultrasound, and in planning the type of therapeutic approach. A multidisciplinary study is essential for the management of these patients from both a clinical and surgical point of view.

Author Contributions All authors participated in the writing of the paper. All authors read and approved the final manuscript.

Funding The authors received no financial sponsors or other funding for this research.

\section{Declarations}

Conflict of interests The authors declare that they have no conflict of interests.

Open Access This article is licensed under a Creative Commons Attribution 4.0 International License, which permits use, sharing, adaptation, distribution and reproduction in any medium or format, as long as you give appropriate credit to the original author(s) and the source, provide a link to the Creative Commons licence, and indicate if changes were made. The images or other third party material in this article are included in the article's Creative Commons licence, unless indicated otherwise in a credit line to the material. If material is not included in the article's Creative Commons licence and your intended use is not permitted by statutory regulation or exceeds the permitted use, you will need to obtain permission directly from the copyright holder. To view a copy of this licence, visit http://creativecommons. org/licenses/by/4.0/.

\section{References}

Papers of particular interest, published recently, have been highlighted as:

- Of importance,

•• Of major importance

1. Bazot M, Bharwani N, Huchon C, et al. European society of urogenital radiology (ESUR) guidelines: MR imaging of pelvic endometriosis. Eur Radiol. 2017;27(7):2765-75. https://doi.org/ 10.1007/s00330-016-4673-z.

2. Nisolle M, Donnez J. Peritoneal endometriosis, ovarian endometriosis, and adenomyotic nodules of the rectovaginal septum are three different entities. Fertil Steril. 1997;68:585-96.

3. Nisenblat V, Bossuyt PM, Farquhar C, Johnson N, Hull ML. Imaging modalities for the non-invasive diagnosis of endometriosis. Cochrane Database Syst Rev. 2016;2(2):CD009591. https://doi.org/10.1002/14651858. CD009591.pub2.

4. Kinkel K, Frei KA, Balleyguier C, Chapron C. Diagnosis of endometriosis with imaging: a review. Eur Radiol. 2006;16(2):285-98. https://doi.org/10.1007/s00330-005-2882-y.

5. Chapron C, Fauconnier A, Vieira M, Barakat H, Dousset B, Pansini V, et al. Anatomical distribution of deeply infiltrating endometriosis: surgical implications and proposition for a classification. Hum Reprod. 2003;18:157-61.

6. Pham M, Sommer C, Wessig C, et al. Magnetic resonance neurography for the diagnosis of extrapelvic sciatic endometriosis. Fertil Steril. 2010;94(1):351.e11-351.e14. https://doi.org/10. 1016/j.fertnstert.2009.12.046.

7. Sampson JA. Peritoneal endometriosis due to menstrual dissemination of endometrial tissue into the peritoneal cavity. Am J Obstet Gynecol. 1927;14:442-69.

8. Chapron C, Marcellin L, Borghese B, Santulli P. Rethinking mechanisms, diagnosis and management of endometriosis. Nat Rev Endocrinol. 2019;15(11):666-82. https://doi.org/10.1038/ s41574-019-0245-z.

9. Vercellini P, Vigano P, Somigliana E, Fedele L. Endometriosis: pathogenesis and treatment. Nat Rev Endocrinol. 2014;10:261-75.

10. Chapron C, Chopin N, Borghese B, et al. Deeply infiltrating endometriosis: pathogenetic implications of the anatomical distribution. Hum Reprod. 2006;21(7):1839-45. https://doi.org/10. 1093/humrep/de1079. 
11. BuckLouis GM, et al. Bisphenol A and phthalates and endometriosis: the endometriosis: natural history, diagnosis and outcomes study. Fertil Steril. 2013;100:162-169.e1-2.

12. Stefansson $\mathrm{H}$, et al. Genetic factors contribute to the risk of developing endometriosis. Hum Reprod. 2002;17:555-9.

13. • Bazot M, Daraï E. Diagnosis of deep endometriosis: clinical examination, ultrasonography, magnetic resonance imaging, and other techniques. Fertil Steril. 2017;108(6):886-94. https://doi. org/10.1016/j.fertnstert.2017.10.026 For the explanation of the imaging features of the different localization of endometriosis.

14. Fauconnier A, Chapron C. Endometriosis and pelvic pain: epidemiological evidence of the relationship and implications. Hum Reprod Update. 2005;11:595-606.

15. Ballard K, Lane H, Hudelist G, Banerjee S, Wright J. Can specific pain symptoms help in the diagnosis of endometriosis? A cohort study of women with chronic pelvic pain. Fertil Steril. 2009;94:20-7.

16. Koninckx PR, Meuleman C, Oosterlynck D, Cornillie FJ. Diagnosis of deep endometriosis by clinical examination during menstruation and plasma CA-125 concentration. Fertil Steril. 1996;65:280-7.

17. Abrao MS, Goncalves MO, Dias JA Jr, Podgaec S, Chamie LP, Blasbalg R. Comparison between clinical examination, transvaginal sonography and magnetic resonance imaging for the diagnosis of deep endometriosis. Hum Reprod. 2007;22:3092-7.

18. • Hindman N, VanBuren W. Imaging spectrum of endometriosis (endometriomas to deep infiltrative endometriosis). Radiol Clin North Am. 2020;58(2):275-89. https://doi.org/10.1016/j.rcl.2019. 11.001 For the explanation of the imaging features of the different localization of endometriosis.

19 Schenken RS. Delayed diagnosis of endometriosis. Fertil Steril. 2006;86(5):1305-6 (Discussion: 1317).

20. Bean E, Naftalin J, Jurkovic D. How to assess the ureters during pelvic ultrasound. Ultrasound Obstet Gynecol. 2019;53(6):729-33.

21. Coutinho A, Bittencourt LK, Pires CE, et al. MR imaging in deep pelvic endometriosis: a pictorial essay. Radiographics. 2011;31:549-67.

22. Exacoustos C, Zupi E, Piccione E. Ultrasound imaging for ovarian and deep infiltrating endometriosis. Semin Reprod Med. 2017;35(1):5-24. https://doi.org/10.1055/s-0036-1597127.

23. Maccagnano C, Pellucchi F, Rocchini L, et al. Diagnosis and treatment of bladder endometriosis: state of the art. Urol Int. 2012;89:249-58.

24. Pateman K, Holland TK, Knez J, et al. Should a detailed ultrasound examination of the complete urinary tract be routinely performed in women with suspected pelvic endometriosis? Hum Reprod. 2015;30:2802-7.

25. Crispi CP, de Souza CA, Oliveira MA, Dibi RP, Cardeman L, Sato $\mathrm{H}$, et al. Endometriosis of the round ligament of the uterus. J Minim Invasive Gynecol. 2012;19:46-51.

26 Hudelist G, Ballard K, English J, et al. Transvaginal sonography vs. clinical examination in the preoperative diagnosis of deep infiltrating endometriosis. Ultrasound Obstet Gynecol. 2011;37:480-7.

27. - Moro F, Leombroni M, Testa AC. Ultrasound Imaging in Endometriosis. Obstet Gynecol Clin North Am. 2019;46(4):643-59. https://doi.org/10.1016/j.ogc.2019.07.004. For the description of the role of US and MRI in the diagnosis of endometriosis

28. Carfagna P, De Cicco NC, De Cicco NA, et al. Role of transvaginal ultrasound in evaluation of ureteral involvement in deep infiltrating endometriosis. Ultrasound Obstet Gynecol. 2018;51:550-5.
29. Maccagnano C, Pellucchi F, Rocchini L, et al. Ureteral endometriosis: proposal for a diagnostic and therapeutic algorithm with a review of the literature. Urol Int. 2013;91(1):1-9.

30. Knabben L, Imboden S, Fellmann B, Nirgianakis K, Kuhn A, Mueller MD. Urinary tract endometriosis in patients with deep infiltrating endometriosis: prevalence, symptoms, management, and proposal for a new clinical classification. Fertil Steril. 2015;103(1):147-52.

31. Noventa M, Saccardi C, Litta P, Vitagliano A, D’Antona D, Abdulrahim B, et al. Ultrasound techniques in the diagnosis of deep pelvic endometriosis: algorithm based on a systematic review and meta-analysis. Fertil Steril. 2015;104:366-83.e2.

32. Holland TK, Cutner A, Saridogan E, Mavrelos D, Pateman K, Jurkovic D. Ultrasound mapping of pelvic endometriosis: does the location and number of lesions affect the diagnostic accuracy? A multicentre diagnostic accuracy study. BMC Womens Health. 2013;13:43.

33. Van den Bosch T, Dueholm M, Leone FP, et al. Terms, definitions and measurements to describe sonographic features of myometrium and uterine masses: a consensus opinion from the Morphological Uterus Sonographic Assessment (MUSA) group. Ultrasound Obstet Gynecol. 2015;46:284-98.

34. Guerriero S, Condous G, Bosch T, et al. Systematic approach to sonographic evaluation of the pelvis in women with suspected endometriosis, including terms, definitions and measurements: a consensus opinion from the International Deep Endometriosis Analysis (IDEA) group. Ultrasound Obstet Gynecol. 2016;48:318-32.

35. Van den Bosch T, de Bruijn AM, de Leeuw RA, et al. A sonographic classification and reporting system for diagnosing adenomyosis. Ultrasound Obstet Gynecol. 2019;53(5):576-82.

36. Reinhold C, Atri M, Mehio A, et al. Diffuse uterine adenomyosis: morphologic criteria and diagnostic accuracy of endovaginal sonography. Radiology. 1995;197:609-14.

37. Kepkep K, Tuncay YA, Goynumer G, et al. Transvaginal sonography in the diagnosis of adenomyosis: which findings are most accurate? Ultrasound Obstet Gynecol. 2007;30:341-5.

38. Di Donato N, Bertoldo V, Montanari G, Zannoni L, Caprara G, Seracchioli R. Question mark form of uterus: a simple sonographic sign associated with the presence of adenomyosis. Ultrasound Obstet Gynecol. 2015;46(1):126-7.

39. Dueholm M. Transvaginal ultrasound for diagnosis of adenomyosis: a review. Best Pract Res Clin Obstet Gynaecol. 2006;20(4):569-82.

40. Meredith SM, Sanchez-Ramos L, Kaunitz AM. Diagnostic accuracy of transvaginal sonography for the diagnosis of adenomyosis: systematic review and metaanalysis. Am J Obstet Gynecol. 2009;201(1):107.e1-107.e6.

41. Luciano DE, Exacoustos C, Albrecht L, et al. Three-dimensional ultrasound in diagnosis of adenomyosis: histologic correlation with ultrasound targeted biopsies of the uterus. J Minim Invasive Gynecol. 2013;20(6):803-10.

42. Patel MD, Feldstein VA, Chen DC, et al. Endometriomas: diagnostic performance of US. Radiology. 1999;210(3):739-45.

43. Jones LP, Morgan MA, Chauhan A. The sonographic spectrum of pelvic endometriosis: pearls, pitfalls, and mimics. Ultrasound Q. 2019;35(4):355-75.

44. Guerriero S, Van Calster B, Somigliana E, et al. Age-related differences in the sonographic characteristics of endometriomas. Hum Reprod. 2016;31:1723-31.

45. Van Holsbeke C, Zhang J, Van Belle V, et al. Acoustic streaming cannot discriminate reliably between endometriomas and other types of adnexal lesion: a multicenter study of 633 adnexal masses. Ultrasound Obstet Gynecol. 2010;35(3):349-53. 
46. Van Holsbeke C, Van Calster B, Bourne T, et al. External validation of diagnostic models to estimate the riskof malignancy in adnexal masses. Clin Cancer Res. 2012;18(3):815-25.

47 Mascilini F, Moruzzi C, Giansiracusa C, et al. Imaging in gynecological disease. 10: Clinical and ultrasound characteristics of decidualized endometriomas surgically removed during pregnancy. Ultrasound Obstet Gynecol. 2014;44(3):354-60.

48. Timor-Tritsch IE, Lerner JP, Monteagudo A, Murphy KE, Heller DS. Transvaginal sonographic markers of tubal inflammatory disease. Ultrasound Obstet Gynecol. 1998;12(1):56-66.

49. Rezvani M, Shaaban AM. Fallopian tube disease in the nonpregnant patient. Radiographics. 2011;31(2):527-48.

50. Chamié LP, Ribeiro DMFR, Tiferes DA, Macedo Neto AC, Serafini PC. Atypical sites of deeply infiltrative endometriosis: clinical characteristics and imaging findings. Radiographics. 2018;38(1):309-28. https://doi.org/10.1148/rg.2018170093.

51. Guerriero S, Alcázar JL, Ajossa S, Pilloni M, Melis GB. Threedimensional sonographic characteristics of deep endometriosis. J Ultrasound Med. 2009;28(8):1061-6.

52. Exacoustos C, Manganaro L, Zupi E. Imaging for the evaluation of endometriosis and adenomyosis. Best Pract Res Clin Obstet Gynaecol. 2014;28(5):655-81.

53. Guerriero S, Ajossa S, Minguez JA, Jurado M, Mais V, Melis GB, et al. Accuracy of transvaginal ultrasound for diagnosis of deep endometriosis in uterosacral ligaments, rectovaginal septum, vagina and bladder: systematic review and meta-analysis. Ultrasound Obstet Gynecol. 2015;46:534-45.

54. Squifflet J, Feger C, Donnez J. Diagnosis and imaging of adenomyotic disease of the retroperitoneal space. Gynecol Obstet Invest. 2002;54:43-51.

55. Reid S, Lu C, Hardy N, et al. Office gel sonovaginography for the prediction of posterior deep infiltrating endometriosis: a multicenter prospective observational study. Ultrasound Obstet Gynecol. 2014;44(6):710-8.

56. Nisenblat V, Prentice L, Bossuyt PM, Farquhar C, Hull ML, Johnson N. Combination of the non-invasive tests for the diagnosis of endometriosis. Cochrane Database Syst Rev. 2016. https://doi.org/10.1002/14651858.CD012281.

57. Guerriero S, Ajossa S, Gerada M, et al. "Tenderness-guided" transvaginal ultrasonography: a new method for the detection of deep endometriosis in patients with chronic pelvic pain. Fertil Steril. 2007;88:1293-7.

58. Valenzano Menada M, Remorgida V, Abbamonte LH, Nicoletti A, Ragni N, Ferrero S. Does transvaginal ultrasonography combined with water-contrast in the rectum aid in the diagnosis of rectovaginal endometriosis infiltrating the bowel? Hum Reprod. 2008;23(5):1069-75.

59. Reid S, Lu C, Casikar I, Reid G, Abbott J, Cario G, et al. Prediction of pouch of Douglas obliteration in women with suspected endometriosis using a new real-time dynamic transvaginal ultrasound technique: the sliding sign. Ultrasound Obstet Gynecol. 2013;41:685-91.

60. Rossi L, Palazzo L, Yazbeck C, et al. Can rectal endoscopic sonography be used to predict infiltration depth in patients with deep infiltrating endometriosis of the rectum? Ultrasound Obstet Gynecol. 2014;43(3):322-7.

61. Guerriero S, Saba L, Ajossa S, Peddes C, Angiolucci M, Perniciano $\mathrm{M}$, et al. Three-dimensional ultrasonography in the diagnosis of deep endometriosis. Hum Reprod. 2014;29:1189-98.

62. Guerriero S, Spiga S, Ajossa S, et al. Role of imaging in the management of endometriosis. Minerva Ginecol. 2013;65:143-66.

63. Del Frate C, Girometti R, Pittino M, et al. Deep retroperitoneal pelvic endometriosis: MR imaging appearance with laparoscopic correlation. Radiographics. 2006;26:1705-18.
64. Saba L, Sulcis R, Melis GB, et al. Endometriosis: the role of magnetic resonance imaging. Acta Radiol. 2015;56(3):355-67. https://doi.org/10.1177/0284185114526086.

65. Nakai A, Togashi K, Kosaka K, et al. Uterine peristalsis: comparison of transvaginal ultrasound and two different sequences of cine MR imaging. J Magn Reson Imaging. 2004;20(3):463-9.

66. - Foti PV, Farina R, Palmucci S, et al. Endometriosis: clinical features, MR imaging findings and pathologic correlation. Insights Imaging. 2018;9(2):149-72. https://doi.org/10.1007/ s13244-017-0591-0. For the description of the role of US and MRI in the diagnosis of endometriosis

67. Bis KG, Vrachliotis TG, Agrawal R, Shetty AN, Maximovich A, Hricak H. Pelvic endometriosis: MR imaging spectrum with laparoscopic correlation and diagnostic pitfalls. Radiographics. 1997;17(3):639-55.

68. Woodward PJ, Sohaey R, Mezzetti TP Jr. Endometriosis: radiologic-pathologic 2001;21(1):193-216.

69. Liang CC, Tsai CC, Chen TC, et al. Management of perineal endometriosis. Int J Gynaecol Obstet. 1996;53:261-5.

70. Donnez J, Nisolle M, Squifflet J. Ureteral endometriosis: a complication of rectovaginal endometriotic (adenomyotic) nodules. Fertil Steril. 2002;77:32-7.

71. Seracchioli R, Raimondo D, Di Donato N, et al. Histological evaluation of ureteral involvement in women with deep infiltrating endometriosis: analysis of a large series. Hum Reprod. 2015;30(4):833-9. https://doi.org/10.1093/humrep/deu360.

72. Chamié LP, Blasbalg R, Pereira RM, Warmbrand G, Serafini PC. Findings of pelvic endometriosis at transvaginal US, MR imaging, and laparoscopy. Radiographics. 2011;31(4):E77-100. https://doi.org/10.1148/rg.314105193.

73. Ghezzi F, Raio L, Cromi A, et al. "kissing ovaries": a sonographic sign of moderate to severe endometriosis. Fertil Steril. 2005;83(1):143-7.

74. Togashi K, Nishimura K, Kimura I. Endometrial cysts: diagnosis with MR imaging. Radiology. 1991;180:73-8.

75. Onbas O, Kantarci M, Alper F, et al. Nodular endometriosis: dynamic MR imaging. Abdom Imaging. 2007;32:451-6.

76. Balaban M, Idilman IS, Toprak H, Unal O, Ipek A, Kocakoc E. The utility of diffusion-weighted magnetic resonance imaging in differentiation of endometriomas from hemorrhagic ovarian cysts. Clin Imaging. 2015;39(5):830-3. https://doi.org/10.1016/j. clinimag.2015.05.003.

77. Foti PV, Ognibene N, Spadola S, et al. Non-neoplastic diseases of the fallopian tube: MR imaging with emphasis on diffusionweighted imaging. Insights Imaging. 2016;7(3):311-27. https:// doi.org/10.1007/s13244-016-0484-7.

78. Siegelman ES, Oliver ER. MR imaging of endometriosis: ten imaging pearls. Radiographics. 2012;32(6):1675-91. https://doi. org/10.1148/rg.326125518.

79. Tham WP, Busmanis I, Tan WC, Kwek JW. Polypoid endometriosis of post vaginal fornix: utility of MRI imaging of pelvis with diffusion weighted imaging for diagnosis. Med $\mathbf{J}$ Malaysia. 2016;71(3):144-6.

80 Gui B, Valentini AL, Ninivaggi V, Marino M, Iacobucci M, Bonomo L. Deep pelvic endometriosis: don't forget round ligaments. Review of anatomy, clinical characteristics, and MR imaging features. Abdom Imaging. 2014;39(3):622-32. https:// doi.org/10.1007/s00261-014-0091-3.

81. Bazot M, Gasner A, Ballester M, Daraï E. Value of thin-section oblique axial T2-weighted magnetic resonance images to assess uterosacral ligament endometriosis. Hum Reprod. 2011;26(2):346-53. https://doi.org/10.1093/humrep/deq336.

82. Medeiros LR, Rosa MI, Silva BR, et al. Accuracy of magnetic resonance in deeply infiltrating endometriosis: a systematic 
review and meta-analysis. Arch Gynecol Obstet. 2015;291(3):611-21. https://doi.org/10.1007/s00404-014-3470-7.

83. Chamié LP, Blasbalg R, Gonçalves MO, Carvalho FM, Abrão MS, de Oliveira IS. Accuracy of magnetic resonance imaging for diagnosis and preoperative assessment of deeply infiltrating endometriosis. Int J Gynaecol Obstet. 2009;106(3):198-201. https://doi.org/10.1016/j.ijgo.2009.04.013.

84. Namimoto T, Awai K, Nakaura T, Yanaga Y, Hirai T, Yamashita Y. Role of diffusion-weighted imaging in the diagnosis of gynecological diseases. Eur Radiol. 2009;19(3):745-60. https:// doi.org/10.1007/s00330-008-1185-5.

85. Caramella $T$, Novellas S, Fournol $M$, et al. Deep pelvic endometriosis: MR imaging features. J Radiol. 2008;89:473-9.
86. Busard MP, van der Houwen LE, Bleeker MC, et al. Deep infiltrating endometriosis of the bowel: MR imaging as a method to predict muscular invasion. Abdom Imaging. 2012;37(4):549-57. https://doi.org/10.1007/s00261-011-9790-1.

87. Yoon JH, Choi D, Jang KT, et al. Deep rectosigmoid endometriosis: B mushroom cap ${ }^{\wedge}$ sign on T2-weighted MR imaging. Abdom Imaging. 2010;35(6):726-31.

88. Balleyguier C, Chapron C, Dubuisson JB, et al. Comparison of magnetic resonance imaging and transvaginal ultrasonography in diagnosing bladder endometriosis. J Am Assoc Gynecol Laparosc. 2002. https://doi.org/10.1016/s1074-3804(05)60099-0.

Publisher's Note Springer Nature remains neutral with regard to jurisdictional claims in published maps and institutional affiliations. 